\title{
A note on a new exponential bound for M-acceptable random variables
}

\author{
Cheikhna Hamallah Ndiaye ${ }^{\dagger}$ and Gane Samb LO $\mathbf{O}^{\ddagger \neq *}$ \\ ${ }^{\dagger}$ LERSTAD, Université de Saint-Louis. LMA-Laboratoire de Mathématiques Appliquées. Université \\ Cheikh Anta Diop, BP. 5005. Dakar-Fann, Sénégal \\ ${ }^{\ddagger}$ LSTA, UPMC, France and LERSTAD, Université de Saint-Louis, Sénégal, BP. 234. Saint Louis \\ Sénégal
}

Received: October 01, 2013; Accepted: November 15, 2013

Copyright (c) 2013, Afrika Statistika. All rights reserved

\begin{abstract}
We present a new exponential inequality as a generalization of that of Sung et al. Sung et al. (2011) for $M$-acceptable random variables, and hence for extended negative ones. Our result is based on the simple real inequality $e^{x} \leq 1+x+(|x| / 2) e^{|x|}, x \in \mathbb{R}$, in place of the following one: $e^{x} \leq 1+x+\left(x^{2} / 2\right) e^{|x|}, x \in \mathbb{R}$, used by many authors. We compare the given bound with former ones.

Résumé. Nous généralisation la notion d'acceptabilité d'une variable aléatoire à celle de $M$-acceptabilité, puis établissons une généralisation de l'inégalité exponentielle de Sung et al. Sung et al. (2011) pour ce type de variables, contenant aussi les variables étendûment négatives. Notre résultat se base sur l'inégalité simple suivante $e^{x} \leq 1+x+(|x| / 2) e^{|x|}, x \in \mathbb{R}$, à la place de celle-ci : $e^{x} \leq 1+x+\left(x^{2} / 2\right) e^{|x|}, x \in \mathbb{R}$, utilisée par plusieurs auteurs. Nous comparons notre borne exponentielle avec d'autres précédemment introduites.
\end{abstract}

Key words: Exponential inequality; Convergence rate; Almost sure convergence; $M$ Acceptable random variables; Negatively associated random variables; Negatively dependent random variables; Extended Negatively dependent random variables; Laplace transform.

AMS 2010 Mathematics Subject Classification : 60F15; 62 G20.

\section{Introduction}

Let $\left\{X_{n}, n \geq 1\right\}$ be a sequence of random variables defined on a fixed probability space $(\Omega, \mathcal{F}, \mathbb{P})$. An exponential inequality for the partial sum $\sum_{i=1}^{n}\left(X_{i}-\mathbb{E}\left[X_{i}\right]\right)$ was introduced

${ }^{*}$ Corresponding author Gane Samb LO: ganesamblo@ganesamblo.net, ganesamblo@yahoo.com Tel +221 3396123 40. Fax +221 96153 53. BP 234 Saint Louis sénégal

Cheikhna Hamallah Ndiaye : ham111266@yahoo.fr 
and proved by Sung et al. (2011) for a class of dependent random variables, that is for $M$ acceptable random variables. This type of inequality provides a measure of the convergence rate for the strong law of large numbers. It is rather classical for the independent case but seems very recent in the dependent case.

We refer to Sung (2009) for an extended introduction on dependent random variables. We will only recall basic definitions to make this paper self-contained. First, recall that a finite family of random variables $\left\{X_{i}, 1 \leq i \leq n\right\}$ is positiveley associated if for any real functions $f_{1}$ and $f_{2}$ defined on $\mathbb{R}^{n}$ and coordinatewise increasing, we have

$$
\operatorname{Cov}\left(f_{1}\left(X_{1}, \ldots, X_{n}\right), f_{2}\left(X_{1}, \ldots, X_{n}\right)\right) \geq 0 .
$$

This type of dependence was introduced in Esary et al. (1967). Next the negative association was set up by Alam and Saxena (1981) and carefully studied by Joag-Der and Proschan (1983). We have negative association whenever for any pair of disjoint subsets $A$ and $B$ of $\{1,2, \ldots, n\}$, with respective cardinalities $p$ and $q$, and for any pair functions respectively defined of $\mathbb{R}^{p}$ and $\mathbb{R}^{q}$, we have

$$
\operatorname{Cov}\left(f_{1}\left(X_{i}, i \in A\right), f_{2}\left(X_{j}, j \in B\right)\right) \leq 0 .
$$

Further, the concept of extended negatively dependent random variables was proposed by Liu (2009) as a generalization of negative dependence introduced by Lehmann (1966), meaning that the family $\left\{X_{1}, \ldots, X_{n}\right\}$ is extended negatively dependent if there is some $M>0$ such that for any real numbers $x_{1}, \ldots, x_{n}$, we have :

$$
\mathbb{P}\left(X_{1} \leq x_{1}, \ldots, X_{n} \leq x_{n}\right) \leq M \prod_{i=1}^{n} \mathbb{P}\left(X_{i} \leq x_{i}\right)
$$

and

$$
\mathbb{P}\left(X_{1}>x_{1}, \ldots, X_{n}>x_{n}\right) \leq M \prod_{i=1}^{n} \mathbb{P}\left(X_{i}>x_{i}\right) .
$$

The special case $M=1$ corresponds to the Lehman original definition. Finally, Sung et al. (2011) defined another negative dependence denamed acceptable for a family $\left\{X_{1}, \ldots, X_{n}\right\}$ for which there exists $\delta>0$ such that for any real $\lambda$ such that $|\lambda| \leq \delta$,

$$
\mathbb{E}\left[\exp \left(\lambda \sum_{i=1}^{n} X_{i}\right)\right] \leq \prod_{i=1}^{n} \mathbb{E}\left[\exp \left(\lambda X_{i}\right)\right] .
$$

Before we go further, we recall that from the steps of Joag-Der and Proschan (1983), that a number of well known multivariate distributions fulfils the negative association property, such as the coordinates of a multinomial vector, that of a multivariate hypergeometric. Also the following stochastic processes satisfy the negative dependence : Dirichlet; permutation distribution; negatively correlated normal distributions; random sampling without replacement and joint distribution of ranks; etc.

Now as to the probability inequality field, as pointed out by Sung et al. (2011), exponential inequalities for positively associated random variables were obtained by Dovroye (1991), Ioannides and Roussas (1999), Oliveira (2005), Sung (2007)), Xing and Yang (2008); Xing 
et al. (2008). We may also cite the following other contributors in this field: Kim and Kim (2007), Nooghabi and Azarnoosh Nooghabi and Azarnoosh (2009), Roussas (1996), Sung (2009), Xing (2009), Xing and Yang (2010), Xing et al. (2009). Many of these inequalities are generalized by Sung et al. (2011), particularly for negatively associated random variables.

In this note we aim at improving significally the current exponential inequalities into two directions. The first is to extend the acceptability of Sung et al. (2011) by $M$-acceptability, for $M>0$, by replacing (1) by

$$
\mathbb{E}\left[\exp \left(\lambda \sum_{i=1}^{n} X_{i}\right)\right] \leq M \prod_{i=1}^{n} \mathbb{E}\left[\exp \left(\lambda X_{i}\right)\right] .
$$

It is clear that extending these inequalities to $M$-acceptable random variable may result in a significant generalization as we point out below. The second generalization concerns the inequality itself. We will see in the next section that our results are based on a much precise real analysis inequality. For example Sung et al. (2011) used the inequality

$$
\forall(x \in R), e^{x} \leq 1+x+\frac{x^{2}}{2} e^{|x|} .
$$

To obtain a better exponential inequality for negative random variables, we will use this inequality

$$
\forall(x \in R), e^{x} \leq 1+x+\frac{|x|}{2} e^{|x|} .
$$

The remainder of this paper is organized as follows. In Section 2, we state our results and make some comments on their generality. The proofs, including that of (4), are given in Section 3. It is worth mentioning that most of the methods of Sung et al. (2011) are reconducted here and hence, will not detailed as it should be.

\section{Results and comments}

\subsection{Results}

As pointed out in the introduction, the inequality (4) is essential. It is used to prove the

Lemma 1. Let $X$ be a random variable with $\mathbb{E} e^{\delta|X|}<\infty$ for some $\delta>0$. Then for any $0<\lambda \leq \delta / 2$,

$$
\mathbb{E}\left[e^{\lambda(X-\mathbb{E} X)}\right] \leq e^{(K \lambda)}
$$

where $K$ is defined as $K=\left(\mathbb{E}\left[|X|^{2}\right]\right)^{1 / 2} \mathbb{E}\left[e^{\delta|X|}\right]$.

This lemma is itself a generalization of in (Lemma 1, Sung et al., 2011). We will get the new exponential inequality from it later.

Theorem 1. Let $\left\{X_{n}, n \geq 1\right\}$ be a sequence of identically distributed $M$ acceptable random variable with $\mathbb{E} e^{\lambda\left|X_{1}\right|}<\infty$ for some $\delta>0$. Then for any $\varepsilon \geq K$,

$$
\mathbb{P}\left(\left|\sum_{i=1}^{n}\left(X_{i}-\mathbb{E}\left[X_{i}\right]\right)\right|>n \varepsilon\right) \leq 2 M \exp (-n \delta / 2(\varepsilon-K)),
$$

where $K=\left(\mathbb{E}\left[\left|X_{1}\right|^{2}\right]\right)^{1 / 2} \mathbb{E}\left[e^{\delta\left|X_{1}\right|}\right]$. 


\subsection{Comments}

\subsubsection{Comparison with Sung et al. results}

For the special case of negatively associated random variables, let us compare our result with those of Sung et al. (2011). In their Remark 2.2, Sung et al. (2011) pointed out that Sung (2009) upper bound and their own one, are of the same convergence rate when $\varepsilon$ is bounded. Our results results based on an unbounded $\varepsilon$ is hence more general than those in the two papers. Next, for $\varepsilon_{n}=2(K \alpha \log n / n)^{1 / 2}, \alpha>0$ and $K=2\left(\mathbb{E}\left[\left|X_{1}\right|^{2}\right]\right)^{1 / 2} \mathbb{E}\left[e^{\delta\left|X_{1}\right|}\right]$, they obtained the bound $2 \exp (-\alpha \log n)$ in their Theorem 2.2. They remarked its supiority to those of Kim and Kim (2007), Nooghabi and Azarnoosh (2009), Xing (2009), Xing et al. (2009) and Xing and Yang (2010). It is clear that our upper bound is better than that of Sung et al. (2011) and then to all the cited ones above.

\subsubsection{Particular case}

By choosing $\varepsilon=n^{\alpha-1}+K$ in Theorem 1, we have the following result

Theorem 2. Let $\left\{X_{n}, n \geq 1\right\}$ be a sequence of identically distributed $M$ acceptable random variables with $\mathbb{E} e^{\delta\left|X_{1}\right|}<\infty$ for some $\delta>0$. Let $\varepsilon_{n}=n^{\alpha-1}+K$, where $\alpha>0$ and $K=\left(\mathbb{E}\left[\left|X_{1}\right|^{2}\right]\right)^{1 / 2} \mathbb{E}\left[e^{\delta\left|X_{1}\right|}\right]$. Then for all $n$,

$$
\begin{gathered}
\left.\mathbb{P}\left(\left|\sum_{i=1}^{n}\left(X_{i}-\mathbb{E}\left[X_{i}\right]\right)\right|>n \varepsilon_{n}\right)\right) \\
\leq 2 M \exp \left(-\frac{\delta}{2} n^{\alpha}\right)
\end{gathered}
$$

Proof. Let $\varepsilon_{n}=n^{\alpha-1}+K$, where $\alpha>0$ and $K=\left(\mathbb{E}\left[\left|X_{1}\right|^{2}\right]\right)^{1 / 2} \mathbb{E}\left[e^{\delta\left|X_{1}\right|}\right]$. Then $\varepsilon_{n}-K \geq 0$ for all $n$. Hence the result follows from Theorem 1 .

\section{Proofs}

Let us begin with the proof of Inequality (4).

\subsection{Proof of (4)}

We first remark that for all $x \in R$,

$$
\frac{|x|}{2} e^{|x|}=\frac{|x|}{2}+\frac{|x|^{2}}{2}+\sum_{n=2}^{\infty} \frac{|x|^{n+1}}{2 n !} .
$$

Next, we remark that for $n \geq 3,2(n-1) ! \leq n(n-1) !=n !$, and then $1 / n ! \leq 1 /(2(n-1) !)$ and next

and finally

$$
\frac{x^{n}}{n !} \leq \frac{|x|^{n}}{n !} \leq \frac{|x|^{n}}{2(n-1) !}
$$

$$
\sum_{n=3}^{\infty} \frac{x^{n}}{n !} \leq \sum_{n=3}^{\infty} \frac{|x|^{n}}{2(n-1) !}=\sum_{n=3}^{\infty} \frac{|x|^{n}}{2(n-1) !}
$$


To conclude, we use the decomposition

$$
e^{x}=1+x+\frac{|x|^{2}}{2}+\sum_{n=3}^{\infty} \frac{|x|^{n}}{2(n-1) !}
$$

and use (5) and (6) to get

$$
\begin{aligned}
e^{x} & =1+x+\frac{|x|^{2}}{2}+\sum_{n=3}^{\infty} \frac{x^{n}}{n !} \leq e^{x} \leq 1+x+\frac{|x|^{2}}{2}+\sum_{n=3}^{\infty} \frac{|x|^{n}}{2(n-1) !} \\
& =1+x+\frac{|x|}{2}\left(|x|+\sum_{n=3}^{\infty} \frac{|x|^{n-1}}{(n-1) !}\right)=1+x+\frac{|x|}{2}\left(|x|+\sum_{n=2}^{\infty} \frac{|x|^{n}}{n !}\right) \\
& =1+x+\frac{|x|}{2}\left(\sum_{n=1}^{\infty} \frac{|x|^{n}}{n !}\right) \leq 1+x+\frac{|x|}{2}\left(\sum_{n=0}^{\infty} \frac{|x|^{n}}{n !}\right)=1+x+\frac{|x|}{2} e^{|x|} .
\end{aligned}
$$

Now let us turn to the proof of Lemma 1.

\subsection{Proof of Lemma 1}

In view of inequality (4) and by using the Hölder inequality for $0<\lambda \leq \delta / 2$, to get

$$
\begin{aligned}
\mathbb{E}\left[e^{\lambda(X-\mathbb{E} X)}\right] & \leq 1+\lambda \mathbb{E}[X-\mathbb{E}[X]]+\frac{\lambda}{2} \mathbb{E}\left[|X-\mathbb{E}[X]| e^{\lambda|X-\mathbb{E} X|}\right] \\
& =1+\frac{\lambda}{2} \mathbb{E}\left[|X-\mathbb{E}[X]| e^{\lambda|X-\mathbb{E} X|}\right] \\
& \leq 1+\frac{\lambda}{2}\left(\left(\mathbb{E}\left[X-\mathbb{E}[X]^{2}\right]\right)\right)^{1 / 2} \mathbb{E}\left[e^{2 \lambda|X-\mathbb{E} X|}\right]^{1 / 2}
\end{aligned}
$$

Now

$$
\left(\mathbb{E}\left[e^{2 \lambda|X-\mathbb{E} X|}\right]\right)^{1 / 2} \leq\left(\mathbb{E}\left[e^{2 \lambda|X+| \mathbb{E} X \mid}\right]\right)^{1 / 2} \leq\left(\mathbb{E}\left[e^{2 \lambda|X|}\right]\right)^{1 / 2}\left(e^{2 \lambda|\mathbb{E} X|}\right)^{1 / 2}
$$

and by Jensen inequality, $e^{2 \lambda|\mathbb{E} X|} \leq \mathbb{E}\left[e^{2 \lambda|X|}\right]$, we end up with

$$
\left(\mathbb{E}\left[e^{2 \lambda|X-\mathbb{E} X|}\right]\right)^{1 / 2} \leq \mathbb{E}\left[e^{2 \lambda|X|}\right]
$$

Next,

$$
\left(\mathbb{E}\left[X-\mathbb{E}[X]^{2}\right]\right)^{1 / 2} \leq\left(\mathbb{E}\left[X^{2}\right]-(\mathbb{E}[X])^{2}\right)^{1 / 2} \leq\left(\mathbb{E}\left[X^{2}\right]\right)^{1 / 2} .
$$

Putting all this together, we get

$$
\mathbb{E}\left[e^{\lambda(X-\mathbb{E} X)}\right] \leq 1+\frac{\lambda}{2}\left(\mathbb{E}\left[X^{2}\right]\right)^{1 / 2} \mathbb{E}\left[e^{2 \lambda|X|}\right] \leq 1+\frac{\lambda}{2} K \leq \exp \left(\frac{\lambda}{2} K\right) .
$$

This achieves the proof. Let us close the proofs section by that of Theorem 1 .

Journal home page: www.jafristat.net 


\subsection{Proof of Theorem 1}

Let $\varepsilon \geq K$. By Markov's inequality, by the definition of $M$ acceptable random variables and by the Lemma, we have for $0<\lambda \leq \delta / 2$,

$$
\begin{gathered}
\mathbb{P}\left(\sum_{i=1}^{n}\left(X_{i}-\mathbb{E}\left[X_{i}\right]\right)>n \varepsilon\right)=\mathbb{P}\left(\exp \left(\lambda \sum_{i=1}^{n}\left(X_{i}-\mathbb{E}\left[X_{i}\right]\right)\right)>\exp (\lambda n \varepsilon)\right) \\
\leq \exp (-\lambda n \varepsilon) \mathbb{E}\left[\exp \left(\lambda \sum_{i=1}^{n}\left(X_{i}-\mathbb{E}\left[X_{i}\right]\right)\right)\right] \\
\leq M \exp (-\lambda n \varepsilon) \prod_{i=1}^{n} \mathbb{E}\left[\exp \left(\lambda\left(X_{i}-\mathbb{E}\left[X_{i}\right]\right)\right)\right] \leq M \exp (-\lambda n \varepsilon) \prod_{i=1}^{n} \exp (K \lambda),
\end{gathered}
$$

since the $X_{i}$ have the same first and second moments. We finally get

$$
\mathbb{P}\left(\sum_{i=1}^{n}\left(X_{i}-\mathbb{E}\left[X_{i}\right]\right)>n \varepsilon\right) \leq M \exp (-\lambda n \varepsilon+\lambda K n) .
$$

The right member is minimum for $\lambda=\delta / 2$ since $\varepsilon \geq K$. We then get for this value

$$
\mathbb{P}\left(\sum_{i=1}^{n}\left(X_{i}-\mathbb{E}\left[X_{1}\right]\right)>n \varepsilon\right) \leq M \exp (-n \delta / 2(\varepsilon-K)) .
$$

Since $\left\{-X_{n}, n \geq 1\right\}$ are also $M$ acceptable random variables, we can replace $X_{i}$ by $-X_{i}$ in the above statement that is ,

$$
\mathbb{P}\left(-\sum_{i=1}^{n}\left(X_{i}-\mathbb{E}\left[X_{i}\right]\right)>n \varepsilon\right) \leq M \exp (-n \delta / 2(\varepsilon-K))
$$

Now observing that $|x|=\max (x,-x)$ and $(|x|>a)=(x>a$ or $x<a)$, we arrive at

$$
\begin{gathered}
\mathbb{P}\left(\left|\sum_{i=1}^{n}\left(X_{i}-\mathbb{E} X_{1}\right)\right|>n \varepsilon\right) \\
\leq \mathbb{P}\left(\sum_{i=1}^{n}\left(X_{i}-\mathbb{E}\left[X_{n}\right]\right)>n \varepsilon\right)+\mathbb{P}\left(-\sum_{i=1}^{n}\left(X_{i}-\mathbb{E}\left[X_{n}\right]\right)>n \varepsilon\right) \\
\leq 2 M \exp (-n \delta / 2(\varepsilon-K)) .
\end{gathered}
$$

\section{References}

Alam, K. and Saxena, K.M.L., 1981. Positive dependence in multivariate distributions. Communications in Statistics Theory and Methods. 10, 1183-1196.

Dovroye, L., 1991. Exponential inequalities in non-parametric estimation, In G. Roussas (Ed.) : Nonparametric functional estimation and related topics. 31-44. Dordrecht : Kluwer Academic Publishers.

Journal home page: www.jafristat.net 
Esary, J.D., Proschan, F. and Walkup, D. W., 1967. Association of random variables, with applications. Annals of Mathematical Statistics, 38, 1466 - 1474.

Ioannides, D.A. and Roussas, G.G., 1999. Exponential inequality for associated random variables. Statistics and Probability Letters, 42, 423-431.

Joag-Der, K. and Proschan, F., 1983. Negative association of random variables with applications. Ann. of Statist. 11, 286-295.

Kim, T.S. and Kim, H.C., 2007. On the exponential inequality for negative dependent sequence. Communications of Korean Mathematical Society, 22, 315-321.

Lehmann, E., 1966. Some concepts of dependence. Annals of Mathematical Statistics. 37, 1137-1153.

Liu, L., 2009. Precise large deviatime for dependent random variables with heany tails. Statist. Probab. Lett. 79(9), 1290-1298.

Nooghabi, H.J. and Azarnoosh, H.A., 2009. Exponential inequality for negatively associated random variables. Statistical Papers, (50), 419-428.

Oliveira, P.E., 2005. An exponential inequality for associated variables. Statistics and Probability Letters. 73, 189-197.

Roussas, G.G., 1996. Exponential probability inequalities with some applications. IMS Lecture Notes Monograph Series. 30, 303-319.

Sung, S.H., 2007. A note on the exponential inequality for associated random variables. Statistics and Probability Letters. (77), 1730-1736.

Sung, S.H., 2009. An exponential inequality for negatively random variables. Journal of Inequalities and Applications, Article ID 64927, 7 pages.

Sung, S.H., Srisuradetchai, P. and Volodin, A., 2011. A note on the exponential inequality for a class of dependent random variables. Journal of the Korean Statistical Society. 40, $109-114$.

Xing, G., 2009. On the exponential inequalities for strictly stationary and negatively associated random variables. Journal of statistical Planning and inference, (139), 3453 3460.

Xing, G. and Yang, S., 2008. Notes on the exponential inequalities for strictly and positively associated random variables. Journal of Statistical Planning and inference, 138, 41324140.

Xing, G. and Yang, S., 2010. An exponential inequalities for strictly stationnary and negatively associated random variables. Communications in Statistics Theory and Methods. 39, $340-349$.

Xing, G., Yang, S. and Lin, A., 2008. Exponential inequalities for positively associated random variables and applications. Journal of Inequalities and Applications. Article ID 385362, 11 pages.

Xing, G. Yang, S. Lin, A. and Wang, X., 2009. A remark on the exponential inequality for negatively associated random variables. Journal of the Korean Statistical Society. 38, 53-57. 